\title{
Simplified Exercise for Mechanical Repositioning of Growing Runners of Forcing-cultured Cucumber Plants against the Supporting Tendril Tensile Strength
}

\author{
Tomonori KAWANO ${ }^{1,2}$, Ayako KAWANO ${ }^{3}$ and Munenori KAWANO ${ }^{3}$ \\ ${ }^{1}$ Faculty of Environmental Engineering, The University of Kitakyushu, \\ Kitakyushu, Fukuoka 808-0135, Japan \\ ${ }^{2}$ University of Florence LINV Kitakyushu Research Center (LINV@Kitakyushu), \\ Kitakyushu, Fukuoka, 808-0134, Japan \\ ${ }^{3} 2159$ Imaizumi-hei, Kiyotake-cho, Miyazaki 889-1206, Japan
}

(Received May 28, 2012; Accepted August 20, 2012)

\begin{abstract}
For cultivation of cucumber (Cucumis sativus L.) under greenhouse, the "wire-suspended cultivation system with intact growing runners" which requires routine exercises for successive repositioning of growing tips of the runners or secondary vines throughout the cultivation period, is now widely employed by the horticultural growers in Japan. During the growth of young vines of cucumber plants under the forcing-culture conditions, repositioning of vines is frequently required due to the spatial limitation or for the ease of fruit harvesting. As the vines are actively self-fixed on the wires by tendril-based networking, the vine repositioning exercise requires cutting or removal of multiple tendrils without severely damaging the vines or growing tips. The present study documented the tendril tensile resisting against the mechanical vine repositioning exercises. The mean tensile strength for single tendril support was shown to be $c a .6 .12 \mathrm{~N}(1.07$ $\mathrm{N} / \mathrm{mm}^{2}$ or $1.07 \mathrm{MPa}$ at the base of tendrils). The force required for tendril removal per single vine would be $5.20 \mathrm{~N}$ and $15.78 \mathrm{~N}$ for exercises performed at 2 day-interval and a week interval, respectively. Based on these figures, we propose here a simplified mechanical procedure for tendril cutting and repositioning of vines, which could be readily automated through the future efforts.
\end{abstract}

Keywords : Cucumis sativus L., forced vine transposition, tendril tensile

\section{INTRODUCTION}

Recent decades, the concept of "plant factories" which aims the automated production of fresh produces chiefly vegetables under precisely controlled environments, has attracted the attentions of engineers, researchers and agriculturists (Ikeda, 2011; Nishida et al., 2012; Nishimura et al., 2012). The prototypic "plant factories" successfully releasing a variety of sprouts, leafy vegetables chiefly lettuce, and tomatoes have been achieved to date (Shimizu et al., 2008; Morimoto, 2005). For expanding the scope of "plant factories" by enabling the automated cultivation of a wide range of agricultural plants, the agricultural and horticultural processes which are successive series of necessary maintaining steps must be translated or re-documented into the automatable protocols based on the records of both plant physiological and mechanical data.

To date, climbing plants chiefly represented by the tendril-bearing plant families such as

Corresponding author: Tomonori Kawano, fax : +81-93-695-3207, e-mail : kawanotom@kitakyu-u.ac.jp 
Vitaceae, Bignoniaceae, Passifloraceae and Cucurbitaceae have not been covered by the trends of "plant factory" developments, possibly due to the belief that mechanical handling of growing vines with position-fixing tendril networks must be achieved manually by experienced specialists. Tendril is a thread-like specialized stem, leaf or petiole used by climbing plants for support and attachment (Liou and Ruan, 2011). As observed by Charles Darwin and Jean-Henri Fabre, the spiral contraction of tendrils occurs after catching the target objects and thus the plants can climb up (Darwin, 1875; Fabre, 1855). In the Cucurbitaceae including cucumber plants (Cucumis sativus L.), tendrils, the coiling organs used for climbing and mechanical support, are part of an axillary bud complex (Gerrath et al., 2008).

During the growth of young vines of cucumber plants under the forcing-culture conditions, repositioning of vines are frequently required due to the spatial limitation or for the ease of fruit harvesting. Especially for cultivation of cucumber plants under greenhouse, the "wire-suspended cultivation system with intact growing runners" which requires routine exercises for successive repositioning of growing tips of the runners or secondary vines throughout the cultivation period, is now widely employed by the horticultural growers in Japan (Kodo et al., 1993). In fact, this system allows the continuous harvesting of cucumber fruits at daily basis for several months by maintaining the growing points at the tip of intact runners. By the downwards repositioning of growing tips of vines at around weekly basis, the regions actively photosynthesizing and producing fruits could be kept suspended at the level suitable for handling. As the vines are actively self-fixed on the wires by tendril-based networking, the vine repositioning exercise requires cutting of multiple tendrils without severely damaging the vines or growing tips.

The present study aims to contribute to the documentation of tendril tensile resisting against the mechanical vine repositioning exercises. Lastly, we propose a simplified mechanical procedure for tendril cutting and repositioning of vines, which could be readily automated through the future efforts.

\section{MATERIALS AND METHODS}

\section{Location and size of the experimental field}

A plastic film-coated greenhouse designed for soil-based forcing culture, sized $730 \mathrm{~m}^{2}$, located in Kiyotakecho, Miyazaki city, Japan, was chosen as the site of experiment. The soil in the test field was Andosol. Nine lanes of soil mound, each maintaining $c a .100$ plants were allocated for protocol efficiency study and the tendril assays.

\section{Plant materials and cultivation history}

Seeds of cucumber plants (Cucumis sativus L., cv. Senshu) and squash root stocks (Cucurbita spp., cv. Yuyuikki-blank) were obtained from Saitama Genshu Ikuseikai Ltd. (Saitama, Japan). Seeds of cucumber were seeded in the sterilized soil on 21st of September, 2011, and maintained under semi-shaded plastic film compartment under sterilized condition. Cucumber seedlings were grafted onto the 1 week-old seedlings of squash grown in the plastic pots with sterilized soil, on 29th of September, 2011. After detaching the cucumber roots (a week after grafting), the resultant grafted seedlings were kept on the plastic pots until re-planted onto the mound of soil in line (seedlings were planted with $40 \mathrm{~cm}$ intervals on the lanes) under greenhouse on 18th of October, 2011. In the first week of November, 2011, the buds at the growing ends of the primary vine at 13-15th nodes were removed and branching and elongation of four secondary vines (key runners numbered from $1^{\prime}$ to $4^{\prime}$, see Fig. 1A) were allowed. The first fruits were harvested on 16 th of November, $2011\left(0.68 \mathrm{~kg}\right.$ fresh weight $\left./ 100 \mathrm{~m}^{2}\right)$. Since then, fruits have been harvested at daily basis until the period of experiments (between 27th of April and 13th of May, 2012). In the spring (April to May, 2012), the productivity of the cucumber was between 8.90 and $14.38 \mathrm{~kg}$ fresh weight/100 $\mathrm{m}^{2}$ at daily basis. Length of one selected secondary vine was shown to be $10.09 \mathrm{~m}$ on the 235 th day 


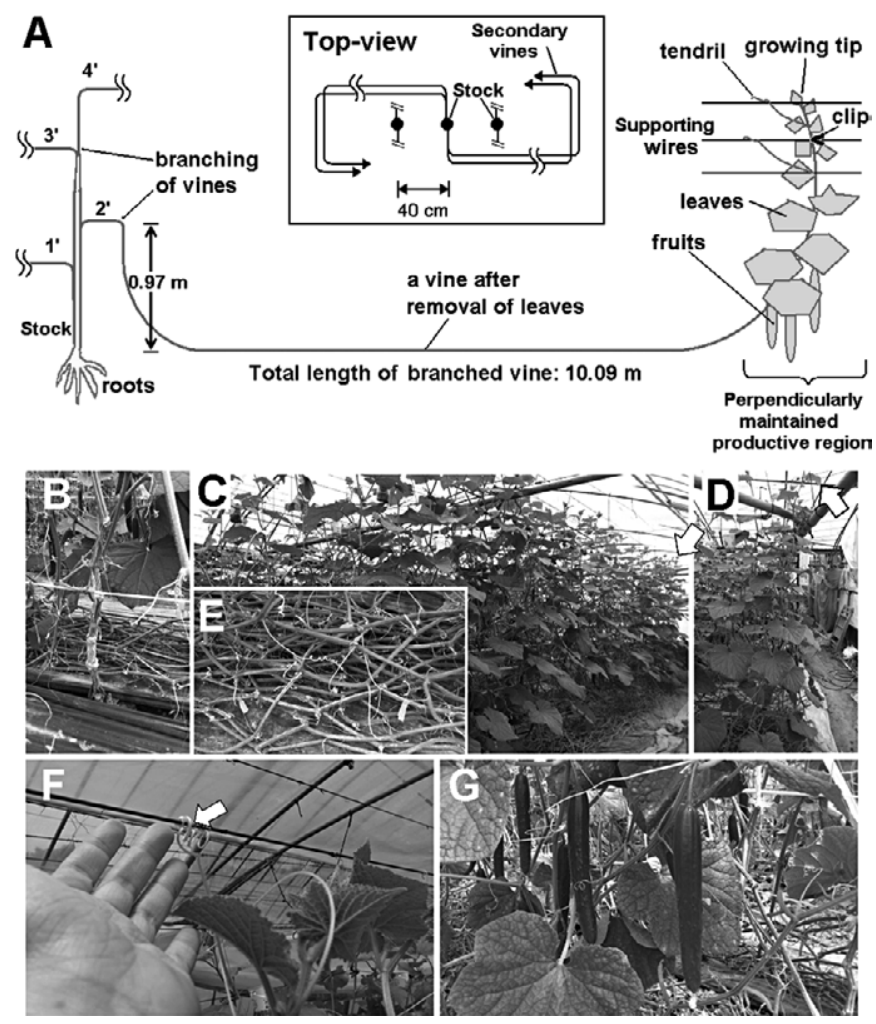

Fig. 1 Forcing-cultivation of cucumber plants under plastic film greenhouse, with the wire-suspended cultivation system leaving the growing tips intact throughout the culture. (A) Orientation of the wiresuspended cucumber plant growth. The inset shows the orientation of four secondary vines allowed to extend anticlockwisely. (B) 8 month-old stock of cucumber plants bearing four secondary vines (numbered from $1^{\prime}$ to $4^{\prime}$ ). (C) A view from the stock (left end) towards the growing tip (arrow). (D) Perpendicularly arranged growing end of the $2^{\prime}$ secondary vine bearing leaves, flowers and fruits, thus referred to as productive region). Growing tip is shown by an arrow. (E) Horizontally arranged bunch of secondary vines simply laid down after removal of leaves. Vine often extended more than $10 \mathrm{~m}$ as an example of measured length is shown in (A). (F) A tendril of young vines tightly linking the plant body and a supporting wire (arrow). (G) Fruits of cucumbers being hanged in the productive region. Tags attached to the vine or the stock were used as visible markers for tracing the origin of single vine.

(13th of May, 2012) after seeding (Fig. 1A).

Repositioning of growing vines

Growers must routinely perform repositioning (lowering) of the tips of growing vines (runners) downwards as illustrated in Fig. 2 during the "wire-suspended cultivation" of cucumber plants. For fixing the newly lowered tips of vines to the supporting wire, clips are commonly used. In order to lower down the growing vines of cucumber, the tendrils attached to the supporting wires must be cut or removed since repositioning of vines without detachment of tendrils often results in unexpected detachment (loss) of growing tips or damaging bending of the young vines. This is actually an annoying task to be performed if the tendrils must be treated one by one individually. However, the network of tendrils can be removed at once simply by applying the downwards-force to the growing vines as suggested by skilled growers (Fig. 2B). In contrast, horizontal force hardly performs the detachment of tendrils but instead the growing tips are readily damaged (Fig. 2C). After removal of the supporting tendrils, the repositioning exercise can be completed by clipping the tip of the vine to the wire at the lower level (Fig. 2D). In the present study, the tendril removal 


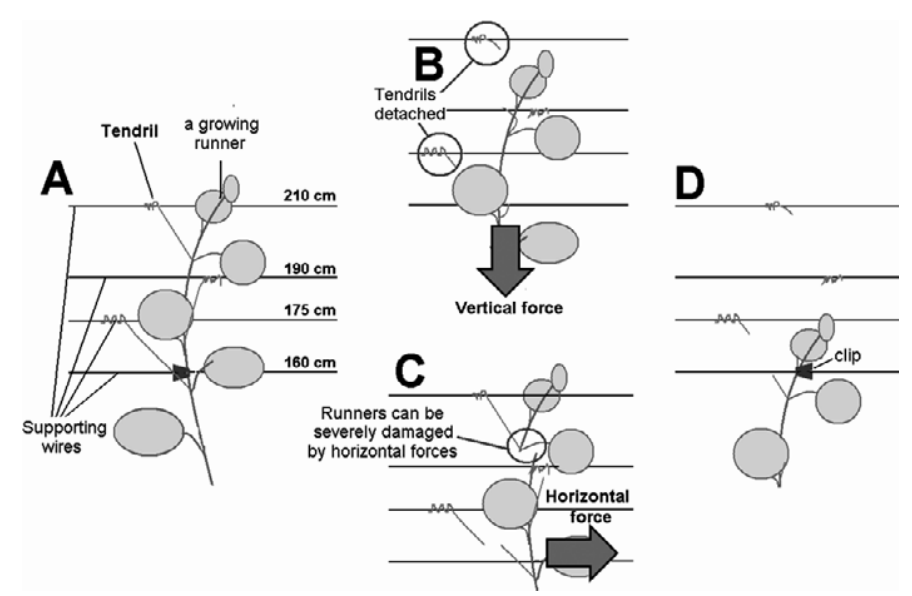

Fig. 2 Tendril detaching and repositioning of growing vines. (A) An overgrown runner (secondary vine) of cucumber supported by the tendrils attached to the supporting wire before the vine reposition is performed. (B) Detachment of tendrils by vertical force (pulling downwards). The clip fixing the plants to the wire must be removed prior to pulling. (C) Failed detachment of tendrils by horizontal force resulting in severe damage to the vine. (D) Clipping of the lowered tip of growing vine at $160 \mathrm{~cm}$ level.

exercise was performed via the path $(\mathrm{A} \rightarrow \mathrm{B} \rightarrow \mathrm{D})$.

Counting of tendrils in task

Counting of the supporting tendrils to be removed or to be detached during the repositioning of growing vines was performed at both the short and long intervals after the previous vine repositioning (i.e. 2 days or 1 week after the previous repositioning exercise). In both cases, 59 individual runners overgrown above the $160 \mathrm{~cm}$ vertical level were examined.

Sampling of tendrils for tensile test

Tendrils were sampled from the vines extending above the wire of $160 \mathrm{~cm}$ level. Both the wire-attached curling tendrils supporting the plant body (Fig. 1F) and freely extending straight tendrils (Fig. 3A) were excised by razor blade from their bases and used for the tensile tests. Adhesive tape was used to fix the tendrils on the tensile unit (plate with a hole) on a spring scale and the tensile strength (measured as gram-force, $\mathrm{gF}$ ) in each tendril was destructively determined by gradually pulling down the base of tendrils until tearing off the tendrils (Fig. 3B).

\section{RESULTS AND DISCUSSION}

\section{Efficiency of the pull-down protocol}

Efficiency of two approaches, namely, with (1) the conventional protocol for removal of individual tendrils followed by repositioning of vines and with (2) the simple pull-down protocol for tendril detachment and vine repositioning at once, were compared by two independent volunteer workers. By performing the conventional approach (on 27th of April, 2012), a single worker routinely completed the repositioning of vines at the rate of 120 vines per hour ( 6 lines with 200 vines, thus, total of 1,200 vines have been processed within $5 \mathrm{~h}$ by 2 workers). The plants treated were examined a week later and the plants were shown to be maintained without being damaged. On the other hand, by the simple pull-down protocol (conducted on 3rd of May, 2012), a single volunteer worker was able to achieve the repositioning of 175 vines per hour (1,400 vines by a single worker in $8 \mathrm{~h}$ ). Again, the plants treated were examined a week later and the plants were shown to be maintained without being damaged. These data roughly suggest that the efficiency of the routines could be enhanced by simplification of procedure by $c a$. $46 \%$. This must be statistically 


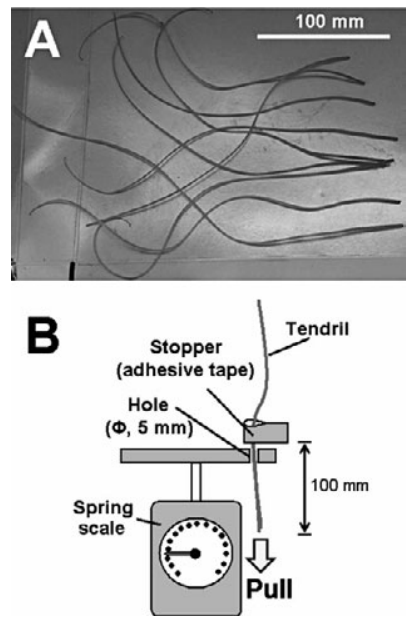

Fig. 3 Procedure for tendril tensile assay. (A) Tendrils excised (to be used immediately). (B) Tests for tensile strength of tendrils at $100 \mathrm{~mm}$-long basal ends were performed as illustrated. For each assay, a tendril with stopper was inserted to the hole on the plate placed above a spring scale. By manually holding and pulling the base of tendrils, vertical (downwards) force was gradually given. Then maximal tensile stress $(\mathrm{gF})$ required for tearing the tendrils were recorded.

examined or confirmed through a large scale demonstration in the future studies.

In addition, it would be less difficult to design the automatable mechanical process for maintenance of growing cucumber vines in the future research since the simple pull-down protocol is much simpler than the conventional protocol. However, in order to achieve this, we need to accumulate the background mechanical data required for handling of the tendril-supported vines of cucumber, especially the data on the dynamics of tendril-based supporting of growing vines.

The following surveys revealed (i) the frequency of tendril detachment per single vine required for vine repositioning exercise and (ii) the mean strength required for treating each vine at two different stages (namely, early and late phases).

Frequency of tendril detachments required during early and late phases of operations

Detachment of wire-attached tendrils from the growing vines or young runners of cucumber plants overgrown above the $160 \mathrm{~cm}$ level was performed by giving vertical force as suggested (Fig. 4). With the protocol with vertical force (pull-down), no vine were de-capped or severely damaged while the tendrils were detached. Mean number of the tendril-detaching events per single vine repositioning action was compared at the early and late phases (Table 1).

With 1 week interval (late phase), the mean height of perpendicularly extending young vines exceeded for over $54 \mathrm{~cm}$ above the $160 \mathrm{~cm}$ level. Through the vine repositioning procedure, 2 to 4 tendrils (mean 2.58 tendrils) were removed or cut. At early phase (2 day-interval) with shorter vines (ca. $29 \mathrm{~cm}$ tall above the $160 \mathrm{~cm}$ level), the tendril detaching events per single vine repositioning action was less than one. As the perpendicularly extending vines were highly fragile if some horizontal force were applied (Fig. 2C), the growers must pay attentions to the connections between different vines via tendril-mediated networking (both the vine-to-tendril attachment and tendril-to-tendril attachment) which might result in unexpected application of horizontal force upon pulling one vine, thus neighboring vine(s) connected via tendril network may be severely damaged. Number of vine-to-vine connections through tendrils was much smaller in the earlier phase group (Table 1), suggesting that the procedure could be much simpler and safer in the early phase, thus suitable for future automation trials. 


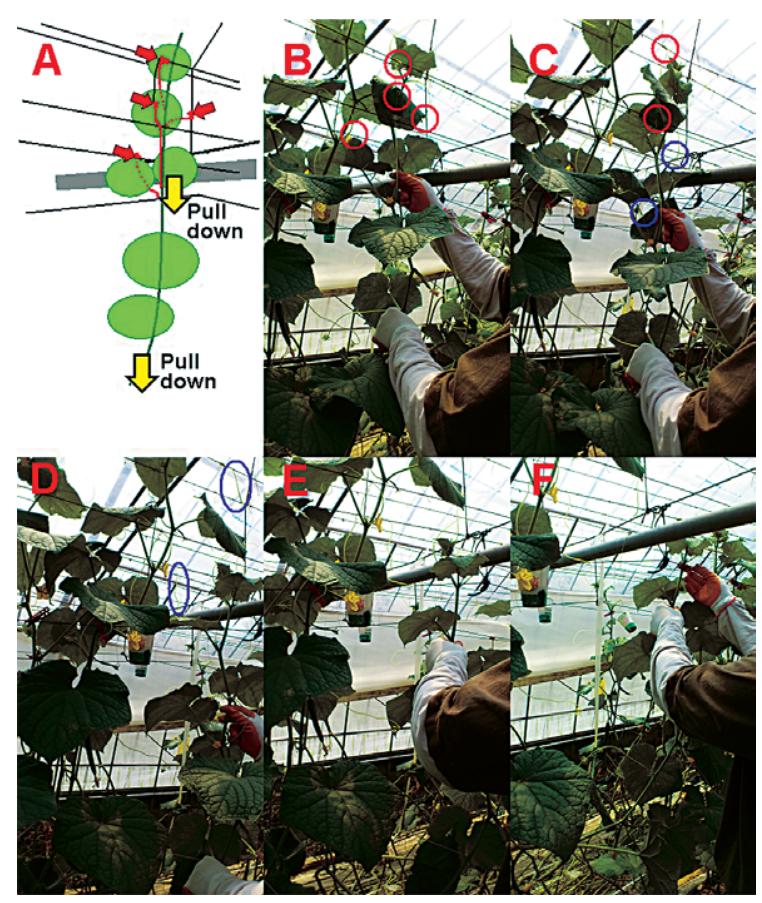

Fig. 4 Tendril detachment by downwards pulling and repositioning of a growing vine performed. (A) Illustration shows the presence wire-attached tendrils (red arrows) supporting the overgrown vines and the direction of the force to be given (arrows). (B-D) Detachment of anchoring tendrils by downward pulling. Red circles highlight the presence of tendrils linking the supporting wire and the vine. Blue circles highlight the tendrils detached from the vine and remaining on the supporting wires. (E, F) Repositioning of the vines at lower position. A clip was used for fixing the vine on the supporting wire at $160 \mathrm{~cm}$ level.

Table 1 Mean number of tendril cutting events per single vine repositioning action during routine maintenance of cucumber's secondary vines.

\begin{tabular}{ccccc}
\hline & $\begin{array}{c}\text { Number of vines } \\
\text { examined }\end{array}$ & $\begin{array}{c}\text { Length of vines } \\
\text { above } 160 \mathrm{~cm} \text { line }\end{array}$ & $\begin{array}{c}\text { Tendril cuts } \\
\text { per vine }\end{array}$ & $\begin{array}{c}\text { Tendrils attached to } \\
\text { neighboring vines }\end{array}$ \\
\hline $\begin{array}{c}\text { Early operation } \\
\text { (at 2 days interval) } \\
\text { Late operation } \\
\text { (at 1 week interval) }\end{array}$ & 59 & $29.3 \pm 6.6$ & $0.85 \pm 0.94$ & $0.08 \pm 0.34$ \\
\hline
\end{tabular}

Independent survey showed that the mean height of vines over the $160 \mathrm{~cm}$ line became $14.7 \pm 4.0 \mathrm{~cm}$, immediately after repositioning operations $(n=59)$. Thus, by early and late operations, the tips of vines were lowered by $c a .14 .6 \mathrm{~cm}$ and $c a .39 .3 \mathrm{~cm}$, respectively.

Tensile strength of tendrils connecting the supporting wire and the vines

Tendril tensile strength was assessed using both freely extending and wire-attached samples (Fig. 5). Among the freely extending tendrils, there were weak tendencies that greater tensile strength could be scored by longer and/or thick tendrils (Fig. 5A, B). The tensile strength of freely extending tendrils and the wire-attached tendrils were compared (Fig. 5C). Interestingly, the tendrils actually suspending or supporting the vines showed significantly higher tensile strength compared to the tendrils newly touching the wire but without actual load of plant bodies including growing leaves and fruits. 

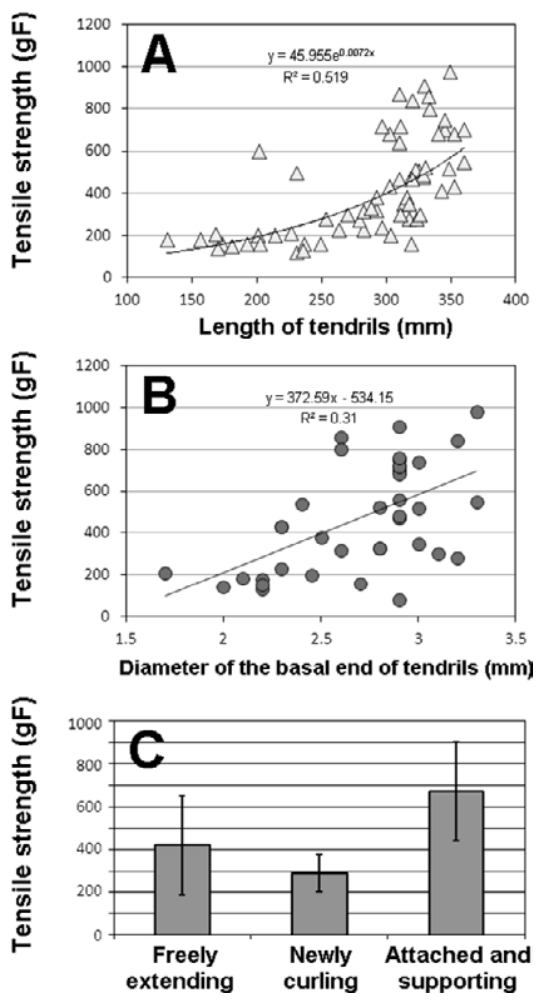

Fig. 5 Tensile strength of tendrils harvested from the growing tips of the secondary vines of cucumber plants. (A) Relationship between the length of freely extending tendrils and the tensile strength recorded. (B) Relationship between the thickness (diameter) of the base of freely extending tendrils and the tensile strength recorded. (C) Comparison of the tensile strength among the tendrils all sampled from the overgrown vines above the $160 \mathrm{~cm}$ level but at different state, namely, freely extending straight tendrils (labeled as "Freely extending"), the tendrils newly curling upon contacts with the supporting wires without load (not suspending the vines; thus labeled as "Newly curling"), and the tendrils attached to the supporting wire and obviously supporting the position of the vines (thus, labeled as "attached and supporting"). Since the tendril samples were all obtained 1 week after the previous vine transposition, both the growing tips and tendrils are no older than 1 week.

\section{Possible strengthening of tendrils upon mechanical stress}

Tensile strength of the plant tissues are highly correlated with the content of cellulose and development of fibers within the tissues (Genet et al., 2005). Borzi (1901) described the presence of gelatinous fibers in cucurbit species and labeled them as the Bianconi plate. These gelatinous fibers were also observed in a cucurbit Bryonia dioca by Kaiser et al. (1994), who added the observation that gelatinous fibers were lignified. Recently, using redvine as a model, Meloche et al. (2007) have demonstrated that gelatinous fibers in tendrils occurred concomitantly with the ability to coil, strongly indicating their role in the coiling process. Furthermore, a microscopic and immunocytochemical survey covering the tendrils and twining vines of a number of species including two cucurbitaceous species showed the presence and distribution of the gelatinous fibers in tendrils and twining veins (Bowling and Vaughn, 2009), further supporting the concept that coiling and twining in vines are caused by the presence of gelatinous fibers. According to above studies (Bowling and Vaughn, 2009; Meloche et al., 2007), tendrils and vines both use gelatinous fibers, but use them in different amounts and positions within the tendril/stem to perform their coiling duties. The most highly coiled structures have the highest densities of gelatinous fibers, whereas 
more gently coiled vines such as morning glory and moonvine have a distribution of gelatinous fibers that are spread throughout the cortical region. It is worth of note that the number and extent of fiber development correlated roughly with the amount of torsion required for the vine to ascend a support (Bowling and Vaughn, 2009) and increases in both xylary and nonxylary fibers and fibrous lignification are inducible by the torsion of the twining stem (Sher et al., 2001). Therefore, it is tempting to test if the touching and coiling events in cucumber tendrils in our system results in the enhancement of fiber development.

\section{Towards development of automated mechanical process}

The mean tensile strength for single tendril support was shown to be $c a .6 .12 \mathrm{~N}(674.5 \pm 232$ $\mathrm{gF}$ ) which could be converted to $1.07 \mathrm{~N} / \mathrm{mm}^{2}$ or $1.07 \mathrm{MPa}$ at the base of tendrils (mean diameter, $2.67 \mathrm{~mm}$ ). Therefore, the force required for tendril removal per single vine would be $5.20 \mathrm{~N}$ and $15.78 \mathrm{~N}$ for early and late phases of operations, respectively. These figures could be the basis for designing the automated mechanical processes.

For automated repositioning of the growing tips of the secondary vines of cucumber, there are two different approaches. One of approaches is the mechanical lowering of structures (such as wires) on which vines are fixed by tendril actions (Hayashi et al., 2002). This approach may be applicable only when the growth of individual vines in line is highly uniform. However, the growth rate of the individual vines may largely differ under natural conditions and therefore repositioning of individual vines (one by one) would be one of the key procedures for finely geared adjustment of vertical positions of fruiting organs. Our present study may contribute to the latter approach. However, further detailed studies are required for manifesting the above approach.

\section{REFERENCES}

Borzi, A. 1901. Anatomia dell'apparo sensomotore dei cirri delle cucurbitacee. Atti. Revev. Accad. Lincei., ser. V, Rc. Cl. Sciencia Floristtes Materiales Naturale 10: 395-400.

Bowling, A. J., Vaughn, K. C. 2009. Gelatinous fibers are widespread in coiling tendrils and twining vines. Am. J. Bot. 96: 719-723.

Darwin, C. 1875. The Movements and Habits of Climbing Plants, John Murray, London.

Fabre, J. H. 1855. De la nature des vrilles des Cucurbitacée. Bull. Soc. Bot. France 2: $512-518$ (Collection of Centre Franco-Japonais d'Histoire des Sciences, Paris-Kitakyushu).

Genet, M., Stokes, A., Salin, F., Mickovski, S. B., Fourcaud, T., Dumail, J.-F., van Beek, R. 2005. The influence of cellulose content on tensile strength in tree roots. Plant Soil 278: 1-9.

Gerrath, J. M., Guthrie, T. B., Zitnak, T. A., Posluszny, U. 2008. Development of the axillary bud complex in Echinocystis lobata (Cucurbitaceae): Interpreting the cucurbitaceous tendril. Am. J. Bot. 95: 773-781.

Hayashi, M., Tsukiji, K., Ezaki, T., Ryu, H. 2002. Climbing plant cultivation unit, cultivation auxiliary tool and climbing plant cultivation method. Japanese Patent (No. JP 2004-89087).

Ikeda, H. 2011. Plant factory. IEEJ Trans. Sens. Micromachin. 131: 200-205.

Kaiser, I., Engelberth, J., Groth, B., Weiler, E. W. 1994. Touch- and methyl jasmonate-induced lignification in tendrils of Bryonia dioca Jacq. Bot. Acta 107: 24-29.

Kodo, H., Yamashita, H., Kaneishi, Y. 1993. Studies on forcing culture of cucumber plants grafted bloomless rootstock I. Influence of lighting and defoliation on growth and yield. (in Japanese) Tokushima Agric. Exper. Sta. Rep. 29: 1-7.

Liou, N.-S., Ruan, G.-W. 2011. The mechanical properties of tendril of climbing plant. Conf. Proc. Soc. Exper. Mechanics Ser. 2: 101-104.

Meloche, C. G., Knox, J. P., Vaughn, K. C. 2007. A cortical band of gelatinous fibers causes the coiling of redvine tendrils: A model based upon cytochemical and immunocytochemical studies. Planta 225: 485498.

Morimoto, T. 2005. Fundamental research for intelligent control in cultivating and storage systems. Environ. Control Biol. 43: 299-303.

Nishida, H., Arima, S., Hatou, K., Takayama, K. 2012. Research centers for intelligent plant production systems in Japan (1) Ehime University. (in Japanese with abstract in English) J. Sci. High Technol Agric. 24: 


\section{CUCUMBER TENDRILS AND VINE REPOSITIONING}

$5^{-9}$.

Nishimura, Y., Fukuda, K., Wada, T. 2012. Research centers for intelligent plant production systems in Japan (2) Osaka Prefecture University. (in Japanese with abstract in English) J. Sci. High Technol. Agric. 24: 1015.

Sher, J. L., Holbrook, N. M., Silk, W. K. 2001. Temporal and spatial patterns of twining force and lignification in stems of Ipomea purpurea. Planta 213: 192-198.

Shimizu, H., Kushida, M., Fujinuma, W. 2008. A growth model for leaf lettuce under greenhouse environments. Environ. Control Biol. 46: 211-219. 